\title{
Propriedades psicométricas da versão brasileira da Escala de Entrincheiramento na Carreira
}

\author{
Mauro de Oliveira Magalhães - Universidade Luterana do Brasil ${ }^{1}$
}

\begin{abstract}
Resumo
A expressão entrincheiramento de carreira significa a imobilização do trabalhador em determinada posição ocupacional devido à percepção de limitação de alternativas de carreira, à evitação da perda de investimentos e a custos emocionais associados à mudança de ocupação. Foram investigadas as características psicométricas de uma versão brasileira da Escala de Entrincheiramento na Carreira. Uma amostra de 668 trabalhadores, 415 homens e 253 mulheres, com idades entre 25 e 65 anos, empregados em organizações públicas, privadas e do terceiros setor, respondeu à escala. Não foram encontradas diferenças de entrincheiramento associadas ao tipo de organização de trabalho. A análise fatorial encontrou os três fatores correspondentes às dimensões propostas para o construto: investimentos de carreira, custos emocionais e limitação de alternativas de carreira. Os indicadores de consistência interna obtidos para a escala total e para cada dimensão de entrincheiramento foram satisfatórios, revelando que todos os itens contribuem para aumentar a precisão do instrumento. As análises indicam aspectos de validade de construto e de precisão satisfatórios para a versão brasileira da Escala de Entrincheiramento na Carreira.

Palavras-chave: Escolha profissional; Trabalho; Psicometria.
\end{abstract}

\section{Psychometric properties of the Brazilian version of the Career Entrenchment Scale}

\begin{abstract}
The expression career entrenchment means the worker's immobilization in a determined occupational position as a result of his/her perception of lack of career alternatives, avoidance of lost of investments and emotional costs associated with occupational changes. The psychometric properties of the Brazilian version of the Career Entrenchment Scale were investigated. A sample of 668 workers, 415 men and 253 women, ages ranging between 25 e 65 years old, employed in public, private ant third sector organizations, answered the scale. Differences of entrenchment were not associated with type of work organization. Factorial analysis found three factors according with the dimensions proposed for the construct: career investments, emotional costs and lack of career alternatives. Internal consistence indicators were satisfactory both for the total scale and each entrenchment dimensions, revealing that all items contribute for higher scale reliability. The analysis showed satisfactory construct validity and reliability aspects for the Brazilian version of the Career Entrenchment Scale.
\end{abstract}

Keywords: Professional choice; Work; Psychometrics.

\section{Introdução}

A turbulência e a incerteza que caracterizam o cenário do mundo do trabalho na atualidade trazem conseqüências importantes para o significado das carreiras profissionais em nossa cultura. Ter uma carreira significou, durante muito tempo, alcançar um posto de trabalho com estabilidade vitalícia, percorrer uma trajetória razoavelmente previsível de promoções e alcançar a aposentadoria por tempo de serviço. $\mathrm{Na}$ atualidade este destino está reservado a uma minoria de trabalhadores inseridos no serviço público. O trabalho é, cada vez mais, uma relação comercial sem comprometimentos de longo prazo. Os trabalhadores tendem a ser remunerados por tarefas, desempenhando múltiplas funções em projetos específicos e temporários. Deste modo, as carreiras tornaram-se mais centradas no indivíduo do que na organização. Neste contexto, divulgam-se estratégias e modelos de gestão de recursos humanos supostamente capazes de tornar os trabalhadores mais empreendedores, criativos e sintonizados com as demandas do mercado (cf. Simonsen, 1997). E, por outro lado, proliferam livros de auto-ajuda com técnicas e conselhos para a autogestão de carreira, encorajando os profissionais a serem autoconfiantes e tolerantes ao risco e a assumirem o controle das próprias carreiras.

A nova realidade das organizações e do mundo do trabalho aumentou a probabilidade de uma estagnação nas carreiras profissionais. Os processos de

\footnotetext{
${ }^{1}$ Endereço para correspondência:

Rua Presidente Kennedy, 84 - Edifício Kennedy - apto. 201 - 40130-200 - Salvador-BA

E-mail: mauro.m@terra.com.br
} 
downsizing reduzem as oportunidades de ascensão para níveis superiores e a obsolescência profissional é um risco sempre iminente. Os conhecimentos, tecnologias e práticas de trabalho renovam-se em ritmo acelerado, e exigem que os sujeitos mobilizem-se continuamente para a manutenção de suas posições mediante a atualização constante de suas credenciais. E com o encurtamento dos lapsos de inovação e ciclos de vida de produtos, essa defasagem na formação profissional dos trabalhadores tende a ocorrer cada vez mais cedo na vida. Portanto, é importante salientar que a obsolescência profissional não é mais o resultado do envelhecimento, mas está relacionada a demandas de aprendizagem não alcançadas ou atendidas, provavelmente em virtude de outros fatores, tais como as habilidades e motivação do sujeito (Zunker, 1994).

Em resposta a estas novas circunstâncias, alguns profissionais têm escolhido criar novos padrões de carreira. Aqueles com recursos suficientes freqüentemente iniciam seus próprios empreendimentos, buscam formação em outras áreas, ou retiram-se da vida profissional para se dedicar a outros interesses. Alguns encontram e desfrutam de oportunidades de crescimento e ampliação do seu perfil profissional, adquirindo maior empregabilidade e autonomia. Outros se aposentam prematuramente e, não raro, mostram baixa satisfação vital. E, por outro lado, há aqueles indivíduos que permanecem imobilizados em suas posições ocupacionais, incapazes ou desinteressados de buscar novas opções (Carson \& Carson, 1997). Esta última condição foi denominada entrincheiramento, para significar um processo de imobilidade na carreira em que o sujeito não apresenta aspectos de adaptabilidade ou motivação para fazer mudanças ocupacionais (Carson, Carson \& Bedeian, 1995).

De acordo com Carson, Carson e Bedeian (1995), o processo de entrincheiramento inclui aspectos como o desejo de evitar o estigma social associado com mudanças na carreira, a busca de preservação da identidade pessoal e profissional, o sentimento de que a idade e a especificidade da formação limitam novas opções, a dificuldade para abdicar do status associado com determinada posição ocupacional e a evitação de perdas financeiras e de investimentos em educação associados com transições de carreira. Observa-se que este conceito surgiu para dar uma resposta a perguntas sobre as razões de trabalhadores que não se identificam com suas atividades de trabalho não mudarem esta situação.

O processo de entrincheiramento foi descrito como iniciando com o acúmulo de investimentos numa área de carreira que, por sua vez, gera custos emocionais no caso de mudanças ocupacionais, que por fim resultam na limitação da percepção de alternativas de carreira. O processo contínuo de investimento e reinvestimento (por exemplo, o treinamento especializado adicional) numa ocupação pode elevar a necessidade do indivíduo de evitar efeitos de dissonância cognitiva, permanecendo na mesma ocupação embora insatisfeito, para não sofrer o desconforto psicológico e de exposição social associado à apresentação de atitudes e comportamentos inconsistentes. Isto é, um processo de preservação psicológica se instala e evolui na medida em que os investimentos se acumulam. E, muitas vezes, a percepção de insatisfação pode levar a mais investimentos, tempo e esforço, na esperança de reverter a situação. O indivíduo tenta provar para si mesmo e para o seu entorno social que fez a escolha certa. Essa preocupação pode prejudicar a capacidade do sujeito para explorar o seu ambiente e identificar alternativas de carreira (Carson \& Carson, 1997; Carson, Carson, Philips \& Roe, 1996).

Sabendo-se que as motivações para se tornar e permanecer entrincheirado podem ser significativas e efetivas para muitos sujeitos, cabe examinar as implicações práticas dessa forma de vínculo com o trabalho e a carreira para indivíduos e organizações. Se profissionais entrincheirados estão resignados a sua situação, é aceitável a hipótese de que isso não trará conseqüências importantes para os mesmos. Porém, os seus empregadores podem se ressentir de uma força de trabalho pouco motivada para a inovação e o crescimento. Em contraste, aqueles profissionais que assistem a suas metas e sonhos profissionais sucumbirem num processo de entrincheiramento, vivem uma situação altamente conflituosa em suas vidas. E essa situação se tornará também problemática para a organização, que necessita do engajamento de seus colaboradores nos projetos empresariais. Por fim, sugere-se que $\mathrm{o}$ desenvolvimento do construto de entrincheiramento e sua investigação são relevantes para o entendimento dos dilemas de carreira dos trabalhadores contemporâneos e para a definição de políticas de gestão de pessoas mais adequadas a este cenário. Nesse sentido, revela-se a necessidade de contar com uma medida adequada deste fenômeno (Carson \& Carson, 1997).

Carson, Carson e Bedeian (1995) propuseram uma medida de entrincheiramento composta de 12 itens e três fatores com quatro itens cada. Nos estudos conduzidos para o desenvolvimento e validação da medida, os 12 itens explicaram 68,5\% da variância. Os autovalores foram 5,06 (fator 1), 1,97 (fator 2) e 1,21 (fator 3). O primeiro fator, denominado investimentos de carreira, explicou $42,1 \%$ da variância, com cargas fatoriais entre 0,53 e 0,77 ; o segundo, denominado custos emocionais, explicou $16,4 \%$, com cargas fatoriais entre 0,70 e 0,84 ; e o terceiro, denominado de limitação de 
alternativas, explicou $10,0 \%$ da variância, com cargas fatoriais entre 0,49 e 0,82 . A dimensão investimentos de carreira pretende significar o tempo, dinheiro e esforço associados com a obtenção de credenciais e habilidades específicas, assim como o salário e a posição hierárquica alcançados. Os custos emocionais referem-se às perdas afetivas associadas com o rompimento de relações interpessoais, perda de status e ameaças à identidade. A limitação de alternativas é a percepção da própria obsolescência, da especificidade de treinamento, da idade, e da falta de atenção ao contexto mais amplo do mundo do trabalho, fatores associados à restrição de oportunidades de crescimento e mudança.

Blau (2001a, 2001b) retomou a medida desenvolvida por Carson, Carson e Bedeian (1995) e propôs a reformulação do construto de três para duas dimensões. A primeira dimensão seria composta por quatro itens, resultando da combinação de dois itens de cada uma das dimensões de custos emocionais e investimentos de carreira, e denominada custos acumulados. A segunda dimensão permaneceu denominada limitação de alternativas, composta de dois itens da escala original. $\mathrm{O}$ autor também fez alterações na redação dos itens e nas alternativas de resposta. Bedeian (2002) replicou à proposta de Blau (2001a, 2001b), questionando a validade de conteúdo dos itens da medida reformulada e considerando que é uma subrepresentação do construto em foco. Bedeian (2002) reanalisou os dados da pesquisa original (Carson, Carson \& Bedeian, 1995), comparando a adequação do modelo bidimensional proposto por Blau (2001a, 2001b) com a medida tridimensional. O autor relatou a inadequação do modelo bidimensional aos dados de sua amostra e reiterou a adequação da formulação original.

A versão brasileira da escala de entrincheiramento de carreira foi utilizada nos estudos de Baiocchi e Magalhães (2004) e Magalhães e Gomes (2005, 2007). Baiocchi e Magalhães (2004) examinaram a relação entre entrincheiramento e comprometimento na carreira, e encontram relação positiva entre a dimensão custos emocionais e a identidade de carreira $(\mathrm{r}=0,25 ; p<0,01)$, uma dimensão do construto comprometimento com a carreira. Esta relação também foi encontrada nos estudos de Magalhães e Gomes (2005, 2007) e confirmam achados de Carson, Carson e Bedeian (1995). A pesquisa de Magalhães e Gomes (2005) revelou que o entrincheiramento está inversamente relacionado a uma medida de generatividade $(\mathrm{r}=-0,14 ; p<0,01)$, principalmente a dimensão limitação de alternativas $(\mathrm{r}=-0,36$; $p<0,01)$. Essa relação confirmou expectativas teóricas, pois a generatividade está ligada à capacidade de criar e oferecer realizações que contribuam para o melhoramento da vida humana, e o entrincheiramento significa a dificuldade do indivíduo para contribuir profissionalmente num espectro mais amplo de atuação, e, conseqüentemente, obter novas possibilidades de generatividade.

Este estudo investigou as propriedades psicométricas da versão brasileira da escala de entrincheiramento de carreira proposta por Carson, Carson e Bedeian (1995), a fim de oferecer aos pesquisadores do comportamento humano no trabalho uma medida adequada desse construto e ampliar a sua utilização em estudos brasileiros. Também foram investigadas diferenças de entrincheiramento associadas ao tipo de organização de trabalho (pública, privada, terceiro setor). O exame desta variável foi considerado relevante tendo em vista o tratamento diferenciado que recebem funcionários públicos em termos de maiores benefícios e garantias de estabilidade no emprego, supostamente tornando mais provável o entrincheiramento.

\section{Participantes}

\section{Método}

Participaram do estudo 668 trabalhadores, sendo $62,12 \%$ homens e $37,88 \%$ mulheres, empregados em organizações da iniciativa privada $(58,23 \%)$, no serviço público $(31,5 \%)$ e em organizações do terceiro setor (cooperativas de trabalhadores) $(10 \%)$, com idades entre 25 e 65 anos (média $=35,2$ ) e com escolaridade mínima de ensino técnico. Todos os participantes tinham mais de 5 anos de carreira profissional, na mesma ou em diversas organizações de trabalho. Os participantes responderam ao instrumento por ocasião da coleta de dados para uma pesquisa mais extensa sobre o desenvolvimento de carreira na vida adulta, cujos resultados pormenorizados podem ser encontrados em Magalhães (2005).

\section{Instrumento}

Foi utilizada uma versão brasileira da Escala de Entrincheiramento na Carreira (EEC) desenvolvida por Carson, Carson e Bedeian (1995). A escala possui 12 itens distribuídos em três fatores com 4 itens cada, a saber: investimentos de carreira, custos emocionais e limitação de alternativas. Os itens são frases que descrevem percepções e sentimentos sobre a possibilidade de uma mudança na carreira profissional. As respostas são assinaladas numa escala Likert de 5 pontos, indicando o grau de concordância com as frases. $\mathrm{O}$ instrumento original, em inglês, foi traduzido para o português, separadamente, por três psicólogos bilíngües. As versões foram comparadas e não mostraram diferenças dignas de nota. A tradução obtida foi revertida para o inglês por outros dois psicólogos bilíngües (backtranslation) a fim de verificar sua equivalência semântica e sintática. Não foram necessários ajustamentos. O estudo 
piloto foi realizado com 32 trabalhadores. Entre eles, 15 estavam matriculados em cursos de pós-graduação nas áreas de administração e engenharia e 17 matriculados em cursos de formação de tecnólogos em gestão de recursos humanos e logística empresarial. $\mathrm{O}$ instrumento mostrou-se de fácil compreensão.

\section{Procedimentos}

A coleta de dados foi coletiva, realizada no local de trabalho ou estudo dos participantes com prévio consentimento das autoridades responsáveis. A pesquisa foi realizada mediante aprovação do Comitê de Ética da instituição de afiliação do pesquisador. Foram atendidas as determinações éticas da resolução 196/1996 do
Conselho Nacional de Saúde (CNS, 1996) sobre a pesquisa com seres humanos.

\section{Análise dos dados}

A validação das dimensões de entrincheiramento foi realizada por medidas de adequação dos dados à análise fatorial, determinação do número de fatores adequados à análise fatorial e verificação da distribuição dos itens em dimensões pelo método de componentes principais. A precisão foi avaliada por meio do alfa de Cronbach. Foi utilizada ANOVA para comparar os escores de entrincheiramento na carreira na dependência do tipo de organização (pública, privada e terceiro setor).

Tabela 1 - Matriz estrutural das dimensões de entrincheiramento na carreira

\begin{tabular}{|c|c|c|c|c|}
\hline \multirow[b]{2}{*}{$\mathrm{N}^{\mathrm{o}}$} & \multirow[b]{2}{*}{ Descrição do item } & \multicolumn{3}{|c|}{ Dimensões } \\
\hline & & IC & FA & $\mathrm{CE}$ \\
\hline 1 & $\begin{array}{l}\text { Se eu ingressasse em outra linha de trabalho/campo de carreira, iria perder muito } \\
\text { investimento em educação e treinamento. }\end{array}$ & 0,668 & & \\
\hline 2 & $\begin{array}{l}\text { Eu tenho muito tempo investido em minha linha de trabalho/campo de carreira, } \\
\text { ficando difícil mudar agora. }\end{array}$ & 0,682 & & 0,356 \\
\hline 3 & Mudar de linha de trabalho/campo de carreira seria muito dispendioso para mim. & 0,705 & & \\
\hline 4 & $\begin{array}{l}\text { Eu já tenho muito dinheiro investido em minha linha de trabalho/campo de carreira } \\
\text { atual, ficando difícil mudar agora. }\end{array}$ & 0,783 & & \\
\hline 5 & $\begin{array}{l}\text { Eu teria várias opções se decidisse por mudar de linha de trabalho/campo de } \\
\text { carreira. }\end{array}$ & & 0,698 & \\
\hline 6 & $\begin{array}{l}\text { Com a minha experiência e histórico profissional, existem alternativas atraentes e } \\
\text { disponíveis para mim em outras linhas de trabalho/campos de carreira. }\end{array}$ & & 0,783 & \\
\hline 7 & $\begin{array}{l}\text { Se eu deixar esta linha de trabalho/campo de carreira, eu me sentirei sem opções } \\
\text { razoáveis para a minha vida profissional. }\end{array}$ & 0,354 & 0,619 & \\
\hline 8 & $\begin{array}{l}\text { Eu percebo alternativas para o meu desenvolvimento profissional se eu quiser fazer } \\
\text { mudanças na minha carreira. }\end{array}$ & & 0,670 & \\
\hline 9 & $\begin{array}{l}\text { Haveria um grande custo emocional envolvido em mudar de linha de } \\
\text { trabalho/campo de carreira. }\end{array}$ & & & 0,682 \\
\hline 10 & $\begin{array}{l}\text { Deixar a minha linha de trabalho/campo de carreira causaria pouco trauma } \\
\text { emocional em minha vida. }\end{array}$ & & & 0,675 \\
\hline 11 & Seria emocionalmente difícil mudar minha linha de trabalho/campo de carreira. & 0,398 & & 0,601 \\
\hline 12 & $\begin{array}{l}\text { Mudar de linha de trabalho/campo de carreira seria fácil do ponto de vista } \\
\text { emocional. }\end{array}$ & & & 0,696 \\
\hline \multicolumn{2}{|r|}{ Eigenvalues } & 3,85 & 1,54 & 1,10 \\
\hline \multicolumn{2}{|c|}{ Porcentagem de variância explicada } & 32,07 & 12,83 & 9,15 \\
\hline
\end{tabular}

\section{Resultados}

Antes de proceder ao cálculo de análise fatorial, a adequação da amostra foi verificada pelo determinante da matriz de correlação, pelo coeficiente de adequação da amostra de Kaiser-Meyer-Olkin (KMO) e pelo teste de esfericidade de Bartlett. O coeficiente Kaiser-MeyerOlkin $(\mathrm{KMO}=0,887)$, o determinante da matriz de correlações $(0,038)$ e o teste de esfericidade de Bartlett $(\chi 2[78, N=668]=2036,15 ; p<0,01)$ revelaram adequação satisfatória dos dados à análise fatorial (Dassa, 1999). Assumindo que as dimensões não são a priori correlacionadas, uma vez que remetem a causas distintas do processo de entrincheiramento, uma análise de componentes principais seguida por rotação varimax foi utilizada para examinar a estrutura fatorial da EEC. O gráfico scree indicou 3 fatores como a melhor explicação possível para os dados apresentados. Três fatores 
obtiveram autovalores adequados (eigenvalue $>1,000$ ) e explicaram mais da metade da variância dos escores de entrincheiramento $(54,07 \%)$. Os autovalores foram 3,85 (fator 1), 1,54 (fator 2) e 1,10 (fator 3). O primeiro fator (IC - investimentos de carreira) explicou 32,07\%, o segundo (LA - limitação de alternativas) explicou 12,83\% e o terceiro (CE - custos emocionais), 9,15\% da variância (Tabela 1).

A análise fatorial revelou que alguns itens saturaram acima de 0,30 (valor mínimo definido para carga fatorial significativa) em mais de um fator. Estes itens $(2,7,11)$ foram mantidos no fator com maior saturação, considerando que a coerência semântica foi atendida em todos os casos e também observando que a magnitude da diferença entre a maior saturação e as demais foi bastante superior a 0,1 (valor mínimo definido para diferença significativa). Os três fatores encontrados agruparam os itens correspondentes às respectivas dimensões do instrumento original e, portanto, revelaram as coerências semânticas pertinentes (Tabela 1).

Os índices de consistência interna (alfas de Cronbach) obtidos para a escala total e para os escores parciais de cada dimensão de entrincheiramento foram os seguintes: 0,82 (EEC); 0,71 (CE - custos emocionais); 0,75 (IC - investimentos de carreira); 0,72 (LA - limitação de alternativas). Foi verificada a contribuição de cada item para a precisão de sua dimensão. Os resultados obtidos mostraram que todos os itens contribuem para aumentar a precisão da sua respectiva dimensão (Tabela 2 ).

Tabela 2 - Alfa de Cronbach por dimensão e se item deletado da escala de entrincheiramento de carreira

\begin{tabular}{|c|c|c|c|c|c|c|c|c|c|c|c|c|c|}
\hline \multirow{2}{*}{ Dimensão } & \multirow{2}{*}{ Alfa } & \multicolumn{12}{|c|}{ Alfa se item deletado } \\
\hline & & 1 & 2 & 3 & 4 & 5 & 6 & 7 & 8 & 9 & 10 & 11 & 12 \\
\hline $\begin{array}{l}\text { Investimentos de } \\
\text { carreira }\end{array}$ & 0,75 & 0,67 & 0,67 & 0,68 & 0,73 & & & & & & & & \\
\hline $\begin{array}{l}\text { Percepção de } \\
\text { alternativas }\end{array}$ & 0,72 & & & & & 0,60 & 0,63 & 0,64 & 0,63 & & & & \\
\hline Custos emocionais & 0,71 & & & & & & & & & 0,59 & 0,58 & 0,55 & 0,63 \\
\hline
\end{tabular}

A análise de variância não revelou diferenças nos escores totais de entrincheiramento na dependência do tipo de organização (pública, privada e terceiro setor), $F(2,668)=0,188, p=0,82$. Análises específicas para cada dimensão de entrincheiramento revelaram que não houve diferenças em limitação de alternativas,
$F(2,668)=0,518, p=0,59$, em investimentos de carreira, $F(2,668)=0,101, p=0,90$, ou custos emocionais, $F$ $(2,668)=0,413, p=0,66$, relacionadas ao tipo de organização. A Tabela 3 apresenta dados de estatística descritiva dos escores de entrincheiramento em cada tipo de organização de trabalho.

Tabela 3 - Médias, desvios padrão e valores de $\mathrm{N}$ para entrincheiramento em cada tipo de organização de trabalho

\begin{tabular}{lccc}
\hline Tipo de organização & N & Média & Desvio-Padrão \\
\hline Privadas & 389 & 38,23 & 9,209 \\
Públicas & 211 & 38,65 & 9,798 \\
Terceiro setor & 68 & 37,92 & 8,708 \\
\hline Total & 668 & 38,35 & 9,358 \\
\hline
\end{tabular}

\section{Discussão}

Os dados colhidos com a escala de Entrincheiramento na Carreira se mostraram adequados ao procedimento da análise fatorial exploratória. $\mathrm{O}$ resultado obtido com o gráfico scree confirmou a estrutura dimensional original de Carson, Carson e Bedeian (1995), recomendando a solução fatorial dos itens a três fatores. Nesse sentido, três fatores obtiveram autovalores adequados e explicaram mais da metade da variância dos escores de entrincheiramento. Portanto, a solução fatorial Psico-USF, v. 13, n. 1, p. 13-19, jan./jun. 2008 mais adequada sugere ser a trifatorial, com resultados desfavoráveis à reformulação proposta por Blau (2001a, 2001b), corroborando a perspectiva de Bedeian (2002).

Além dos três fatores explicarem uma parte importante da variância total, todos os itens da EEC saturaram nas dimensões para as quais foram elaborados. Embora alguns itens tenham saturado acima de 0,30 em mais de um fator, todos os itens revelaram saturações mais elevadas no fator definido pelo modelo, de forma a permitir que a validade de construto (via análise fatorial exploratória) seja assumida. 
A avaliação dos índices de consistência interna da escala total e de cada dimensão de entrincheiramento revelou resultados positivos, com níveis de precisão totais e parciais satisfatórios. Todos os itens contribuíram para a precisão da sua respectiva dimensão, indicando a relevância da sua permanência no instrumento.

A ausência de relação entre entrincheiramento e o tipo de organização de trabalho (pública, privada e terceiro setor) sugere que o construto está mais associado a aspectos disposicionais do que contextuais relacionados aos comportamentos de carreira. Pode-se pensar que as características de instabilidade e incerteza que cercam as condições de trabalho e emprego na atualidade façam emergir com mais força e nitidez o impacto das diferenças individuais nas atitudes e comportamentos dos profissionais em relação as suas carreiras. Se a estrutura ocupacional estável do passado não exigia a iniciativa e a criatividade em alto grau, o cenário emergente demanda o incremento da iniciativa e do planejamento independente da vida e da carreira. E considerando que a restrição de alternativas adequadas de trabalho seja um fato irredutível para muitos, esses indivíduos podem ser impelidos a criar uma estrutura de vida própria que faça conviver papéis profissionais pouco satisfatórios com atividades familiares, comunitárias e de lazer que resultem num saldo positivo de realização pessoal. Nessa perspectiva, pode-se dizer que, ao invés de um declínio das forças disposicionais, num contexto geral de imprevisibilidade esses aspectos se tornem mais relevantes para o entendimento dos comportamentos de carreira.

A escala de entrincheiramento na carreira tem sido utilizada em amostras brasileiras e tem revelado associações teoricamente relevantes com outras variáveis, tais como o comprometimento organizacional, o comprometimento com a carreira, motivação vital, generatividade e interesses vocacionais (Baiocchi \& Magalhães, 2004; Magalhães \& Gomes, 2005; Magalhães \& Gomes, 2007). Portanto, sugere ser um instrumento adequado à realidade brasileira e recomenda-se o seu uso em pesquisas que relacionem o construto a outros fatores e processos de interesse.

\section{Referências}

Baiocchi, A. \& Magalhães, M. (2004). Relações entre processos de comprometimento, entrincheiramento e motivação vital em carreiras profissionais. Revista Brasileira de Orientação Profissional, 5(1), 63-71.

Bedeian, A. G. (2002). Issues in the dimensional structure of career entrenchment. Journal of Occupational and Organizational Psychology, 75, 247-250.
Blau, G. (2001a). Testing the discriminant validity of occupational entrenchment. Journal of Occupational and Organizational Psychology, 74, 85-93.

Blau, G. (2001b). On assessing the construct validity of two multidimensional constructs: Occupational commitment and occupational entrenchment. Human Resource Management Review, 11, 279-298.

Carson, K. D. \& Carson, P. P. (1997). Career entrenchment: A quiet march toward occupational death? Academy of Management Review, 11(1), 63-75.

Carson, K. D., Carson, P. P. \& Bedeian, A. G. (1995). Development and construct validation of a career entrenchment measure. Journal of Occupational and Organizational Psychology, 68, 301-320.

Carson, K. D., Carson, P. P., Phillips, J. S. \& Roe, C. W. (1996). A career entrenchment model: Theoretical development and empirical outcomes. Journal of Career Development, 22(4), 273-286.

Conselho Nacional de Saúde (1996). Resolução para pesquisa envolvendo seres bumanos. Resolução 196/1996, Brasília.

Dassa, C. (1999). Analyse multidimensionnelle exploratoire et confirmative. Montreal: Université Montréal.

Magalhães, M. O. (2005). Personalidades vocacionais e desenvolvimento na vida adulta: generatividade e carreira profissional. Tese de doutorado. Porto Alegre, RS: Universidade Federal do Rio Grande do Sul Instituto de Psicologia, Programa de Pós-Graduação em Psicologia.

Magalhães, M. O. \& Gomes, W. B. (2005). Personalidades vocacionais, generatividade e carreira na vida adulta. Revista Brasileira de Orientação Profissional, 6, 7179.

Magalhães, M. O. \& Gomes, W. B. (2007). Personalidades vocacionais e processos de carreira na vida adulta. Psicologia em Estudo, 12(1), 95-103.

Simonsen, P. (1997). Promoting a development culture in your organization: using career development as a change agent. Palo Alto, CA: Davies-Black.

Zunker, V. G. (1994). Career counseling: Applied concepts of life planning. Pacific Grove, CA: Brooks/Cole.

Recebido em janeiro de 2008

Reformulado em maio de 2008

Aprovado em junho de 2008 
Sobre o autor:

Mauro de Oliveira Magalhães é psicólogo, doutor em Psicologia pela Universidade Federal do Rio Grande do Sul (UFRGS), professor adjunto no Curso de Psicologia da Universidade Luterana do Brasil (ULBRA), pesquisador visitante no Programa de Pós-Graduação em Psicologia da Universidade Federal da Bahia (UFBA) (bolsista FAPESB) e career coach (Certificado pelo Career Planning and Adult Development Network - EUA). 